\title{
Isolated pulmonary vein atresia
}

\author{
S Cullen, P F Deasy, E Tempany, D F Duff
}

\section{Department of Paediatric Cardiology, Our Lady's Hospital for Sick Children, Dublin $S$ Cullen D F Duff \\ Department of Paediatrics, Our Lady's Hospital for Sick Children, Dublin P F Deasy E Tempany \\ Correspondence to Dr D F Duff, Department of Cardiology, Our Lady's Hospital for Sick Children,
Crumlin, Dublin 12, Ireland. Accepted for publication} 18 December 1989

\begin{abstract}
Two patients with isolated atresia of the pulmonary veins are described. One patient presented with haemoptysis and the other with recurrent unilateral chest infections. The diagnosis was confirmed in both instances by cardiac catheterisation and pulmonary angiography. One patient died one year after the diagnosis was made and the second child has been stable during a follow up period of three years.
\end{abstract}

Isolated atresia of the pulmonary veins is a very rare congenital anomaly. To date 16 patients without additional congenital heart disease have been reported in English. ${ }^{1-3}$ Recurrent haemoptyses and chest infections are the typical presenting features. Without treatment mortality approaches $50 \%$ in the reported cases. ${ }^{1}$ Pulmonary angiography is the definitive investigation. We report two additional patients with pulmonary vein atresia without additional congenital heart disease.

\section{Case reports}

CASE 1

A one year old boy was admitted to another hospital for investigation of melena and suspec-

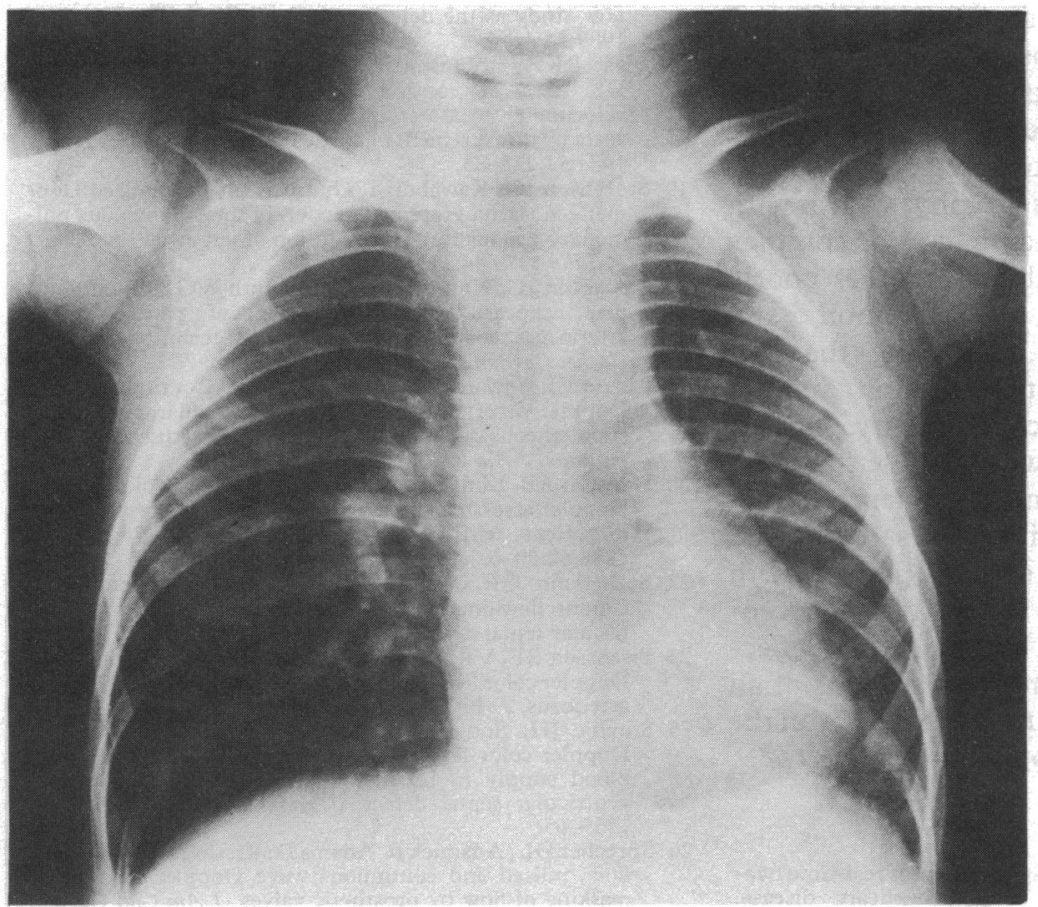

Figure 1 This chest $x$ ray in patient 1 suggests reduced volume of the left lung because of the slight mediastinal shift to the left and the crowding of the ribs on that side. Arterial vascular markings are decreased within the left lung. ted haematemesis. The infant had been well up to one month before admission. A full blood count showed a haemoglobin concentration of $70 \mathrm{mg} / 1$. A chest $x$ ray showed left upper lobe pneumonia. Treatment included a blood transfusion before referral.

At admission to our hospital the child was pale, but otherwise physical examination was normal. A full blood count showed a haemoglobin concentration of $81 \mathrm{mg} / \mathrm{l}$. Coagulation studies were normal. A barium swallow and follow through did not show any abnormality in the upper gastrointestinal tract but did show a retro-oesophageal right subclavian artery. The chest $x$ ray showed reduced lung volume of the left lung and reduced pulmonary markings on that side with deviation of the trachea to the left (fig 1).

On day 9 after admission the patient was feverish and tachypnoeic. There was intercostal recession with reduced air entry in the left lung and bilateral crepitations. A chest $x$ ray showed extensive bilateral pneumonia. Treatment with ampicillin and gentamicin was started. A throat swab gave a heavy growth of Branhamella catarrhalis. On day 19 after admission he had a fresh haemoptysis (not a haematemesis) witnessed by the attending nurse. No resuscitation was required.

A ventilation perfusion scan showed $95 \%$ perfusion to the right lung and 5\% to the left lung. The ventilation perfusion ratio in the left lung was 19:1. An electrocardiogram showed mild right ventricular hypertrophy with an incomplete right bundle branch block pattern. Cardiac catheterisation was performed and a pulmonary angiogram was obtained (table). All right heart pressures including the right pulmonary arterial wedge pressure were normal. It was not possible to obtain a left pulmonary arterial wedge pressure and the left pulmonary veins could not be entered from the left atrium. Angiography showed significant underperfusion and hypoplasia of the proximal and distal pulmonary arteries in the left lung as well as in the right upper lobe (fig 2A). There was extremely slow passage of contrast through the left lung after a selective left pulmonary angiogram with no evidence of normal pulmonary veins (fig 2B). Contrast that had refluxed into the right pulmonary artery filled the left atrium through normal pulmonary veins at a time when the perfusion of the left lung was still in the late arterial phase. The venous drainage from the left lung eventually occurred to the azygos vein (fig $2 \mathrm{C}$ ), probably via the bronchial venous system, though obstructed pulmonary venous drainage to this site 
Haemodynamic data in two patients with isolated pulmonary vein atresia

\begin{tabular}{|c|c|c|c|c|}
\hline \multirow[b]{2}{*}{ Site } & \multicolumn{2}{|l|}{ Case 1} & \multicolumn{2}{|l|}{ Case 2} \\
\hline & Pressure ( $m m \mathbf{H g}$ ) & $\mathrm{O}_{2}$ saturation $(\%)$ & Pressure ( $\mathrm{mm} \mathrm{Hg}$ ) & $\mathrm{O}_{2}$ saturation $(\%)$ \\
\hline $\begin{array}{l}\text { Superior vena cava } \\
\text { Right atrium } \\
\text { Right ventricle } \\
\text { Main pulmonary artery } \\
\text { Right pulmonary artery } \\
\text { Left pulmonary artery } \\
\text { Right pulmonary arterial wedge } \\
\text { Left pulmonary arterial wedge } \\
\text { Left ventricle } \\
\text { Femoral artery }\end{array}$ & $\begin{array}{l}\bar{M}_{\text {ean }}=0 \\
25 / 5 \\
25 / 13 \text { (mean: } 17) \\
23 / 12 \text { (mean:16) } \\
\overline{\text { Mean }}=2 \\
\overline{70} / 4 \\
-\end{array}$ & $\begin{array}{l}78 \\
77 \\
74 \\
75 \\
75 \\
- \\
- \\
- \\
94\end{array}$ & $\begin{array}{l}\overline{M e a n}=2 \\
23 / 4 \\
22 / 13(\text { mean: } 16) \\
23 / 12 \text { (mean:18) } \\
20 / 12(\text { mean: } 16) \\
\text { Mean }=23 \\
\text { Mean }=10 \\
-116 / 57\end{array}$ & $\begin{array}{l}79 \\
81 \\
79 \\
89 \\
94 \\
85 \\
- \\
- \\
96\end{array}$ \\
\hline
\end{tabular}

could not be excluded. On the right side the venous drainage from the upper lobe was not defined. This suggested that an abnormality, resembling that in the left lung also affected the right lung. The dominant pulmonary flow on the right side was to the middle and the lower lobes with normal venous drainage to the left atrium.

A left pneumonectomy was recommended; however, the child's guardians refused operation, preferring a conservative approach. The child was discharged home 7 weeks after admission. During the next year, he was admitted to the referring hospital with pneumonia on one occasion. At a review examination at our hospital one year after the initial assessment his guardians did not report any problems and declined intervention. One week later he was admitted to the referring hospital with a 4 day history of dyspnoea, loss of appetite, and lethargy. He was dyspnoeic and pale with evidence of pneumonia. Radiology showed extensive pulmonary opacities and cardiomegaly. Pneumonia and congestive cardiac failure were diagnosed and he was treated with digoxin, diuretics, and antibiotics. Despite intensive treatment he died 5 days after admission. Permission for necropsy was refused.

CASE 2

A 7 year old boy presented with a history of recurrent right sided chest infections with wheeze that had been present since he was 22 months old. He had about three attacks a year. Each episode lasted several weeks and was slow to respond to antibiotics and or bronchodilators.

When he was six and a half years old he was admitted to another hospital with an extensive right sided pneumonia that failed to resolve
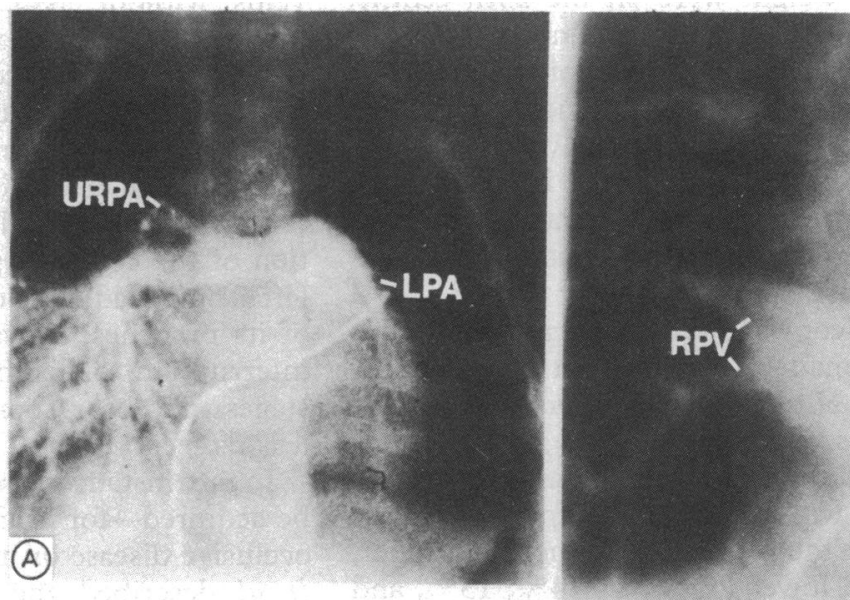

(B)

Figure 2 (A) In patient 1 the pulmonary angiogram showed decreased perfusion and hypoplasia of the pulmonary arteries within the left lung ( $L P A)$ and the right upper lobe (URPA) and preferential filling of the right middle and lower lobe; $(B)$ delayed passage of contrast through the left lung with normal pulmonary veins (RPV) on the right side; and (C) poorly defined concentration of contrast in the azygos vein $(A Z)$ in the late phase of left lung perfusion.

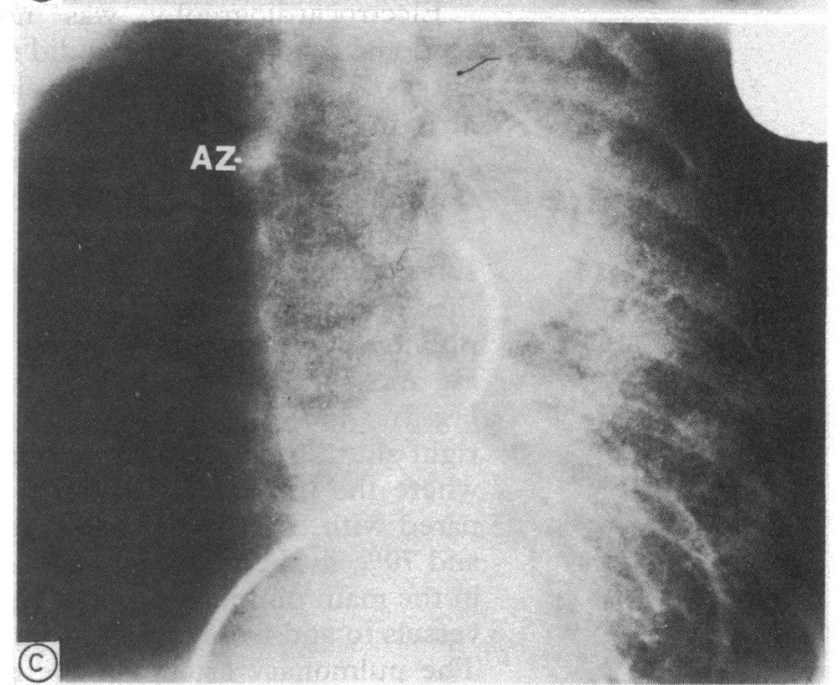




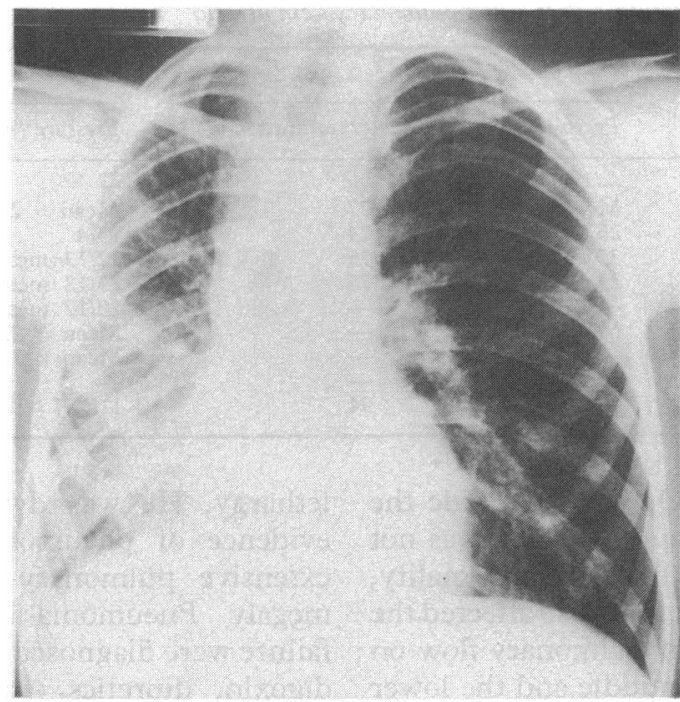

Figure 3 Chest $x$ ray in patient 2 showing reduced volume of the right lung with mediastinal displacement to the right and elevation of the right hemidiaphragm. Interstitial markings in the right lower lobe were increased and there was infiltration in the middle lobe.

despite antibiotic treatment and physiotherapy. When he was referred to our hospital, physical examination showed tracheal deviation to the right, decreased percussion, and decreased air entry in the right lung base with areas of bronchial breathing. Cardiac examination was normal and in particular there was no evidence of pulmonary hypertension. His height and weight were on the 25 th centile. Chest $x$ ray showed reduced volume of the right lung with extensive consolidation of the middle and lower lobes. There was a mediastinal shift to the right with some elevation of the diaphragm on that side (fig 3). In view of the experience with case 1 pulmonary venous atresia complicated by pneumonia was suspected.

Bronchoscopy showed a large amount of pus in the right main bronchus, from which a heavy growth of Haemophilus influenzae was cultured. An isotope scan showed no detectable perfusion to the right lung. Ventilation in the right lung was reduced. Computer analysis showed that the ventilation to the right upper, middle, and lower lobes was $14 \%, 13 \%$, and $3 \%$ respectively.

Electrocardiography was normal. Cross sectional echocardiography did not display the entry of the right pulmonary veins into the left atrium and so cardiac catheterisation and a pulmonary angiogram were performed when the pneumonia had resolved (table).

As in the first patient the right heart pressures were normal. In this patient, however, there was a discrepancy between the mean right pulmonary arterial wedge pressure of $23 \mathrm{~mm}$ $\mathrm{Hg}$ and a mean left wedge pressure of $10 \mathrm{~mm}$ $\mathrm{Hg}$. In addition, there was evidence of a left to right shunt within the right pulmonary artery where the oxygen saturation was $94 \%$ compared with $85 \%$ in the left pulmonary artery and $79 \%$ in the right ventricle. An angiogram in the main pulmonary artery showed that the vessels to and from the left lung were normal. The pulmonary artery on the right side was smaller than normal and it mainly supplied the right middle lobe. There was a double bulge on the right side of the left atrium where the right pulmonary veins should have entered (fig 4A). A selective angiogram of the distal right pulmonary artery showed that the pulmonary veins that were defined ended blindly in a varicose dilatation at the right hilum (fig 4B).

The remainder of the blood flow to the right lung was supplied by bronchial collateral arteries arising from the descending aorta and the right subclavian artery (fig 4C). Blood flowed retrogradely through bronchial to pulmonary anastomoses into the right pulmonary artery and from there to the left pulmonary artery (fig 4D). This gave rise to the high oxygen saturation in the right pulmonary artery (table). Venous drainage from the right lung was via the bronchial venous system into the systemic venous system.

Although a pneumonectomy was considered he was treated conservatively and has remained free of important respiratory infections over the past three years. There was a concern that he might develop a scoliosis after the pneumonectomy at this age and it was felt that this would be less likely to occur if operation could be deferred until his pubertal growth spurt was complete.

\section{Discussion}

Congenital atresia of individual pulmonary veins without associated structural abnormalities of the heart is a rare anomaly. To date, there have been reports of 16 cases in English. ${ }^{1-3}$ Patients usually present with recurrent pulmonary infections or haemoptyses.

Congenital atresia of the pulmonary veins is thought to be caused by defective incorporation of the common pulmonary vein into the left atrium leading to obstruction in some or all of its branches. Edwards has postulated that interruption of embryogenesis at different stages may be responsible for the variety of lesions seen. ${ }^{4}$

In rare instances the venous obstruction may be acquired-for example secondary to venoocclusive disease or mediastinitis. Shrivastava et al described the unusual association of unilateral pulmonary venous atresia with pulmonary veno-occlusive disease in the contralateral lung in a 7 year old patient who died. ${ }^{3}$ There was no associated congenital heart defect in this patient. Beerman et al reported a patient in whom pulmonary venous obstruction did not develop until some years after ductal ligation, after normal pulmonary venous drainage had been seen before operation. Beerman $e t$ al did not suggest a possible cause for the obstruction. ${ }^{5}$

The typical presenting features were noted in both of our patients. In patient 1 an initial impression of haematemesis was changed when an episode of haemoptysis was witnessed. Recurrent chest infections with wheezing prompted referral of the second patient. In both instances radiology showed the reduced volume of the affected lung; this sign was reported in earlier reports. Smaller hilar vessels 

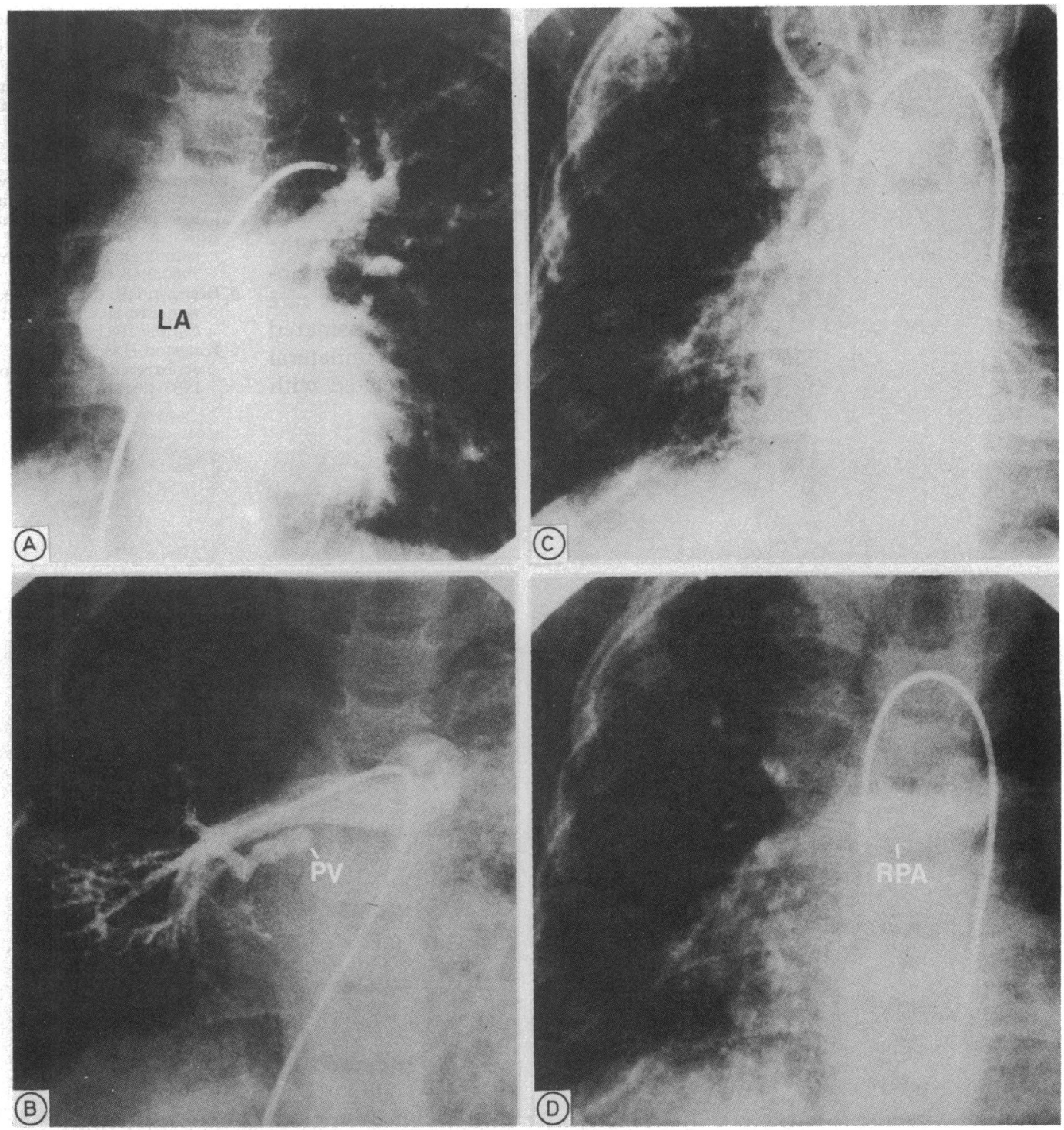

Figure 4 (A) Late phase of main pulmonary arteriogram in patient 2 showing normal pulmonary venous drainage of the left lung and absent right pulmonary veins with bulges on the right lateral wall of the left atrium $(L A)$. (B) Selective distal right pulmonary arteriogram showing blind ending varicose right pulmonary vein $(P V)$. (C) Late filling of bronchial and systemic collateral arteries within the right lung after the aortogram. (D) Retrograde filling of the right pulmonary artery $(R P A)$ from bronchial arteries via bronchopulmonary anastomoses.

on the affected side as seen in our two patients have also been reported before. ${ }^{6}$ A ventilation perfusion scan suggested the diagnosis in the first patient because it showed the almost total absence of perfusion with diminished ventilation in the affected lung and in the second patient the scan supported the clinical impression of pulmonary vein atresia.

Many patients with pulmonary vein atresia have severe pulmonary hypertension, though as in our two patients the pulmonary artery pressure may be normal. In the first patient it was impossible to obtain a satisfactory wedge pressure on the left side and attempts to enter the pulmonary veins on that side also failed. In the case of the second patient the wedge pressure was increased in the affected lung. We assume that the increased blood flow through the bronchial arteries which normally carry less than $5 \%$ of the cardiac output and communicate with the pulmonary branches via precapillary anastomoses was responsible for the raised pulmonary wedge pressure and the elevated pulmonary arterial oxygen saturation that was found on the affected side.

Pulmonary angiography confirmed the diagnosis in each case. Selective injections into the pulmonary arteries showed slow passage of contrast into the affected lung with failure to demonstrate pulmonary veins in one patient and definition of a single small varicose vein with a blind ending in the other. Drainage from the affected lung was through the bronchial venous system in both instances, though we cannot exclude the possibility that obstructed pulmonary venous pathways drained the left lung to the azygos vein in the first patient. We believe that pulmonary venous drainage is perhaps less likely because there was no increase in the oxygen saturation in the superior vena cava.

Without surgical treatment mortality in this condition approaches $50 \%{ }^{1}$ Surgical treatment mainly comprises pneumonectomy, because a direct approach on the pulmonary veins is usually not feasible. In the patient who 
died we cannot be certain that a left pneumo- haemoptysis and radiographic evidence of nectomy would have achieved a satisfactory long term result because of the apparent involvement of the pulmonary vein from the right upper lobe. In the surviving patient, we shall perform pneumonectomy if his clinical condition worsens.

We describe two patients with congenital atresia of the pulmonary veins and confirm the value of isotope scans and pulmonary angiography in clarifying the diagnosis of this rare condition. The diagnosis should be considered in any child with recurrent unilateral pneumonia, especially if it is associated with

reduced lung volume on the affected side.

1 Cabrera A, Vazquez C, Leukona I. Isolated atresia of the left pulmonary veins. Int J Cardiol 1985;7:298-302.

2 Reid JM, Jamieson MPG, Cowan MD. Unilateral pulmonary vein stenosis. $B r$ Heart $J$ 1986;55:599-601

3 Shrivastava S, Moller JH, Edwards JE. Congenita unilateral pulmonary venous atresia with pulmonary veno-occlusive disease in contralateral lung: an unusual association. Pediatr Cardiol 1986;7:213-9.

4 Edwards JE. Congenital stenosis of pulmonary veins: pathologic and developmental considerations. Lab Invest 1960;9:46-66.

5 Beerman LB, Oh KS, Park SC, et al. Unilateral pulmonary vein atresia: clinical and radiographic spectrum. Pediatr vein atresia: clinical and

6 Kingston HM, Patel RG, Watson GH. Unilateral absence or extreme hypoplasia of pulmonary veins. Br Heart $J$ 1983;49:148-53. 